\title{
Implementation of VLSI interconnect design
}

\author{
D.Sathyanarayanan $^{1 *}$ and M.Mohamed ${ }^{2}$ \\ Department of Computer Science, Faculty of Informatics, University of Gondar, Ethiopia ${ }^{1}$ \\ Department of Information Technology, Faculty of Informatics, University of Gondar, Ethiopia ${ }^{2}$
}

(C2018 ACCENTS

\begin{abstract}
One of the key problems in VLSI interconnect design is the topology construction of signal nets with the minimum cost. The Steiner tree problem is to find the tree structure which connects all pins of the signal net such that the wire length (i.e., cost) can be minimized. If all edges of the tree are restricted to the horizontal and vertical directions as are the case in VLSI design, the problem is called rectilinear Steiner tree (RST).The problem of optimizing interconnections between microelectronic devices is an evolving area under VLSI architectures. Steiner tree is a fundamental problem in the automatic inter-connects optimization for VLSI design. Existing methodologies using a Steiner tree approach are not optimal in terms of path length.
\end{abstract}

Keywords

Rectilinear, Steiner, Graph, Topology and VLSI.

\section{Introduction}

The rectilinear Steiner tree (RST) problem is defined as follows: Given a set (say $\mathrm{T}$ ) of terminals in the Cartesian plane, find a shortest interconnection of the terminals using only horizontal and vertical lines. Lines are allowed to meet at points other than the terminals; non-terminal meeting points are called Steiner points. In general, the RST can contain, in addition to the pins of the net, some more points are called Steiner points. In particular, the RST without Steiner points is called rectilinear minimum spanning tree (RMST) studied by Hwang [1].

This study provides a probabilistic analysis for the rectilinear Steiner tree problem. By considering all possible topologic structures connecting every pair of pins, the probabilities of the structures passing over individual edges are calculated. The optimal Steiner tree under statistical sense is the tree with maximum sum of the probabilities for all edges of which the tree is comprised [2]. Experiment shows that the obtained tree topology is very close to the optimal RST. Steiner Trees Can be applied in Networks such as Communication networks, Water pipes or heating ducts in buildings. Applications in VLSI design such as wire routing phase, minimization of wiring, signal propagation time, and/or capacitance.

*Author for correspondence

The goal is designing an algorithm to find the shortest possible interconnectivity between any two devices employing rectilinear Steiner trees. In Rectilinear Steiner minimal tree (RSMT) the distances are measured by the rectilinear metric (horizontal and/or vertical for the plane, orthogonal for higher dimension space.). The other types of Steiner trees are Euclidean \& Graph based.

\section{A. Grid}

Consider a set of $\mathrm{N}$ points, $\mathbf{P}=\left\{\mathrm{p}_{1}, \mathrm{p}_{2}, \ldots, \mathrm{p}_{\mathrm{n}}\right\}$ in a plane, where the location of $p_{i}$ is denoted by $\left(x_{i}, y_{i}\right)$. Assuming $x_{i} \neq x_{j}$ and $y_{i} \neq y_{j}$ for all $i \neq j$. We construct a grid, which consists of the intersections (or, segments) of horizontal and vertical lines through all points. It was shown in [3] that only those segments within the smallest rectangle enclosing all points need to be considered in obtaining the RST.

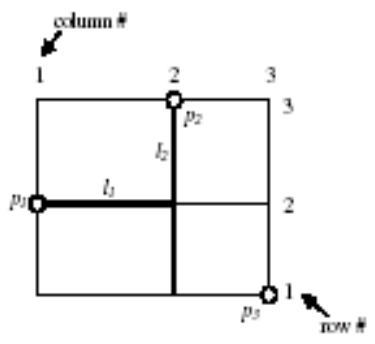

Figure 1 The grid graph for a set of three points

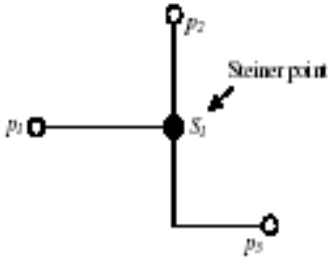

Figure 2 an optimal steiner tree of figure 1 
An optimal RST is a subset of segments, $\mathbf{T}$, such that $\mathbf{T}$ is a tree for given points and the total wire length over all segments in $\mathbf{T}$ is minimum. Figure 1 illustrates the grid for a set of three points, $\mathbf{P}=\left\{\mathrm{p}_{1}\right.$, $\left.\mathrm{p}_{2}, \mathrm{p}_{3}\right\}$. An optimal Steiner tree of Figure 1 is shown in Figure 2, where $S_{1}$ is a Steiner point. If we number the columns and rows of the grid graph, the symbol $\mathrm{H}(\mathrm{i}, \mathrm{j})$ can be used to represent the horizontal segment which lies on row $i$ and between columns $j$ and $j+1$. Similarly, we use $V(i, j)$ to represent the vertical segment which lies on column $j$ and between rows $\mathrm{i}$ and $\mathrm{i}+1$. For instance, the segments $l_{1}$ and $l_{2}$ in Figure 1 can be denoted by $\mathrm{H}(2,1)$ and $\mathrm{V}(2,2)$, respectively. Note that the rows are numbered from bottom to top in the graph, and the columns are numbered from left to right.

\section{Literature survey}

Several effective heuristic approaches have been proposed towards the optimal or sub-optimal solutions for Steiner trees, Hanan [2] showed an optimal algorithm when the net contains no more than four pins. Hwang [4] proved that the ratio of tree lengths between an RMST and an RST is no worse than 3/2. An $\mathrm{O}(\mathrm{N} \log \mathrm{N})$ algorithm for the RST was also proposed in [5], while the results were far from the optimal solution. A good survey on Steiner tree problems can be found in [6]. For a comprehensive survey of the interconnect design, the readers are referred to $[7,8]$.

A new graph convexity was introduced, arising from Steiner intervals in graphs that are a natural generalization of geodesic intervals [9].In general, the RST can contain, in addition to the pins of the net, some more points are called Steiner points. In particular, the RST without Steiner points is called rectilinear minimum spanning tree (RMST) studied by Hwang [1].

\section{Proposed work}

The model is explained with help of the Figure 3. A grid is constructed with six points in the plane. The vectors $\mathrm{H}, \mathrm{V}, \mathrm{HD}, \mathrm{VD}$ are computed as follows, The values of $\mathrm{H}, \mathrm{V}, \mathrm{HD}, \mathrm{VD}$ for the Figure 3 are $\mathrm{H}=\{2,6,5,1,3,4\}, \mathrm{V}=\{1,2,3,4,5,6\}, \mathrm{HD}=\{2,3,1,7,1\}$

and $\mathrm{VD}=\{2,2,1,1,5\}$ respectively.

Where

$\mathrm{H}$-gives the row number of the points in the plane.

$\mathrm{V}$-gives the column number of the points in the plane.

HD-gives the horizontal differences.

VD-gives the vertical differences.

$\mathrm{n}, \mathrm{k}$ vertical grid distance

97
$1, \mathrm{~m}$ horizontal grid distance

Consider two points $\mathrm{p}_{3}, \mathrm{p}_{5} \in \mathrm{P}$ in the grid as shown in Figure 3.

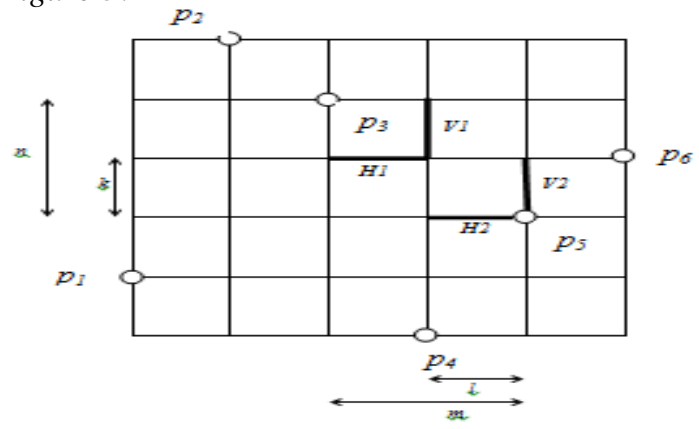

Figure 3 Mathematical model

We assume $\mathrm{H}(5)<\mathrm{H}(3)$ and $\mathrm{V}(3)<\mathrm{V}(5)$. The horizontal and vertical distances between $p_{i}$ and $p_{j}$ are $\mathrm{m}=\mathrm{v}(\mathrm{j})-\mathrm{v}(\mathrm{i})$, and $\mathrm{n}=\mathrm{h}(\mathrm{i})-\mathrm{h}(\mathrm{j})$ respectively. Let $\mathrm{M}$ be the number of all possible shortest paths from $\mathrm{p}_{3}$ to $\mathrm{p}_{5}$. The number of those paths which pass through the segment $\mathrm{H}(\mathrm{i}, \mathrm{j}-1)$ (i.e., $\mathrm{H}_{2}$ in Figure 3 ) only depends on $\mathrm{m}$ and $\mathrm{n}$, and is denoted by $\mathrm{F}(\mathrm{m}, \mathrm{n})$. The number of those paths which pass through the segment V(I, J) (i.e., $\mathrm{V}_{2}$ in Figure 3) is also a function of $\mathrm{m}$ and $\mathrm{n}$, denoted by $G(m, n)$. Obviously, we have $M=F(m, n)$ $+\mathrm{G}(\mathrm{m}, \mathrm{n})$.

From a statistical point of view, the probability of a shortest path between the two points passing through $H(i, j-1)$ is given by $F(m, n) / M$.

More generally, let us consider the specific horizontal segment $\mathrm{H}(\mathrm{I}+\mathrm{k}, \mathrm{J}-\mathrm{l}-1)$ where 1 is 0 in Figure $3 . \mathrm{k}$ varies from 0 to $\mathrm{n}$ and $\mathrm{l}$ varies from 0 to $\mathrm{m}-1$. Among the $\mathrm{M}$ shortest paths from $\mathrm{p} 3$ to $\mathrm{p} 5$ the number of paths that pass through this segment $\mathrm{H} 2$ is given by $\mathrm{F}(\mathrm{m}-\mathrm{l}, \mathrm{n}-\mathrm{k}) \cdot \mathrm{F}(\mathrm{l}+1, \mathrm{k})$.

Thus, the probability of the shortest path passing through this segment is expressed as

$\mathrm{PH}(\mathrm{I}+\mathrm{k}, \mathrm{J}-\mathrm{l}-1)=\frac{\mathrm{F}(\mathrm{m}-1, \mathrm{n}-\mathrm{k}) \cdot \mathrm{F}(1+1, \mathrm{k})}{\mathrm{F}(\mathrm{m}+1, \mathrm{n})}$

Similarly, the probability of a shortest path between p3 and p5 passing through the vertical segment V2 is expressed as

$P V(I+k, J-1)=\underline{G(m-1, n-k) \cdot G(1, k+1)}$ $\mathrm{G}(\mathrm{m}, \mathrm{n}+1)$

\section{Proposed Algorithm}

Step 1: Given $\mathbf{P}=\left\{\mathrm{p}_{1}, \mathrm{p}_{2}, \ldots, \mathrm{p}_{\mathrm{n}}\right\}$, construct its grid. For $\mathrm{i}=1,2, \ldots, \mathrm{n}$; 
Step 2: Compute the horizontal and vertical differences between pair of points,

Step 3: Compute $F(m, n)$ and $G(n, m)$ for $m=1,2$, $\ldots, \mathrm{N}$, and $\mathrm{n}=0,1, \ldots, \mathrm{N}-1$;

$$
\begin{aligned}
F(m, n) & =\Sigma_{k} F(m-1, k), & & k=0 \text { to } n \\
G(m, n) & =\Sigma_{k} G(m-1, k), & & k=1 \text { to } n
\end{aligned}
$$

Step 4: Obtain the probability matrices, $\mathbf{P H}$ and $\mathbf{P V}$, using equations (1) and (2) for all $\mathrm{N}(\mathrm{N}-1) / 2$ pairs of points;

Step 5: Construct a tree $\mathbf{T}$ by selecting the segments one by one in the decreasing order of their corresponding probabilities in $\mathbf{P R}$ and $\mathbf{P C}$, and performing the following three operations during the selection:

1) Ignore the current segment if selecting it would leads to a loop which contradicts the tree definition;

2) Delete all redundant segments once all points in $\mathbf{P}$ have been connected by the tree;

3) Calculate the total wire length of the obtained. Steiner tree after the selection is completed.

\section{Experimental result}

Applying the algorithm (B), for the problem and the result obtained is shown below: The following figure shown in Figure 4 gives the construction of final tree for the above graph.

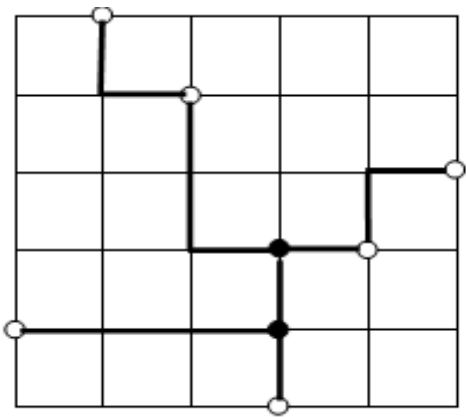

Figure 4 Final tree

\section{Conclusion}

Probability analysis of Steiner tree is studied in detail. The solution has been obtained for the given set of points. The result obtained using the algorithm is 30; whereas the result obtained for the same example in [1] were 32. The results are found to be better than those by the previous technique or very close to the optima. The approach applied is a heuristic one, better and new advanced algorithm techniques can be implied to improve the result and efficiency.

\section{Acknowledgment}

None.

\section{Conflicts of interest}

The authors have no conflicts of interest to declare.

\section{References}

[1] Hwang FK. An O (n $\log n$ ) algorithm for rectilinear minimal spanning trees. Journal of the ACM. 1979; 26(2):177-82.

[2] Hanan M. On Steiner's problem with rectilinear distance. SIAM Journal on Applied Mathematics. 1966; 14(2):255-65.

[3] Mandoiu II, Vazirani VV, Ganley JL. A new heuristic for rectilinear Steiner trees. IEEE Transactions on Computer-Aided Design of Integrated Circuits and Systems. 2000; 19(10):1129-39.

[4] Hwang FK. On Steiner minimal trees with rectilinear distance. SIAM Journal on Applied Mathematics. 1976; 30(1):104-14.

[5] Hwang FK, Richards DS. Steiner tree problems. Networks. 1992; 22(1):55-89.

[6] Tang Y, Yang W, Guo T. Definition and algorithms for reliable Steiner tree problem. Journal of Systems Science and Complexity. 2015; 28(4):876-86.

[7] Cong J, He L, Koh CK, Madden PH. Performance optimization of VLSI interconnect layout. Integration, the VLSI Journal. 1996; 21(1-2):1-94.

[8] Bienkowski M, Kraska A, Schmidt P. A deterministic algorithm for online Steiner tree leasing. In workshop on algorithms and data structures 2017 (pp. 169-80). Springer, Cham.

[9] Gologranc T. Graphs with 4-Steiner convex balls. Taiwanese Journal of Mathematics. 2015; 19(5):132540.

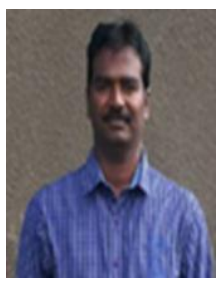

D. Sathyanarayanan has completed his Bachelor degree in Computer science and Engineering at University of Madras in 1998 and Master in Engineering (CSE) at Anna University on 2004. At present doing Research degree in the relevant discipline at AMET university in Chennai, Tamil nadu.

Email: sathya.d.77@gmail.com

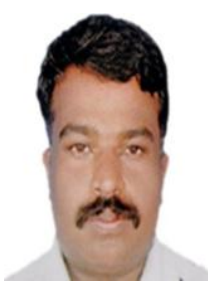

M. Mohamed obtained his Bachelor in Physics degree at M.S.S Wakf Board College in Madurai during the academic year 1995-1998, Master in Computer Applications at Jamal Mohamed College during the Academic Year 1998-2001 and his research degree in Computer Science at Periyar University on 2012. He has teaching experience almost $16+$ years. 